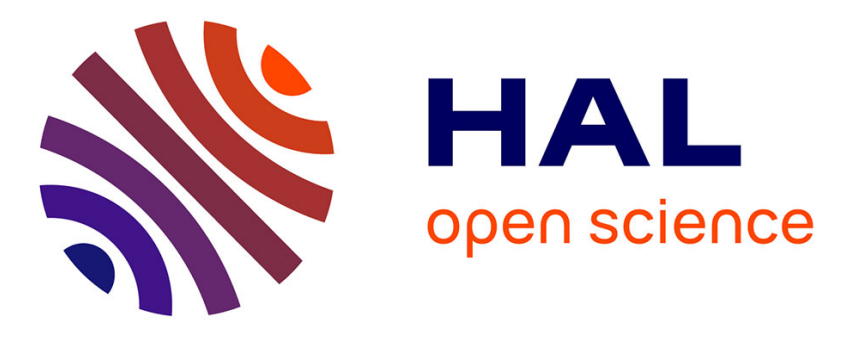

\title{
Robust dynamic hand gesture interaction using LTE terminals
}

Weiyan Chen, Kai Niu, Deng Zhao, Rong Zheng, Dan Wu, Wei Wang, Leye Wang, Daqing Zhang

\section{- To cite this version:}

Weiyan Chen, Kai Niu, Deng Zhao, Rong Zheng, Dan Wu, et al.. Robust dynamic hand gesture interaction using LTE terminals. IPSN 2020: 19th ACM/IEEE International Conference on Information Processing in Sensor Networks, Apr 2020, Sydney (online), Australia. pp.109-120, 10.1109/ipsn48710.2020.00017 . hal-03002712

\section{HAL Id: hal-03002712 \\ https://hal.science/hal-03002712}

Submitted on 12 Nov 2020

HAL is a multi-disciplinary open access archive for the deposit and dissemination of scientific research documents, whether they are published or not. The documents may come from teaching and research institutions in France or abroad, or from public or private research centers.
L'archive ouverte pluridisciplinaire $\mathbf{H A L}$, est destinée au dépôt et à la diffusion de documents scientifiques de niveau recherche, publiés ou non, émanant des établissements d'enseignement et de recherche français ou étrangers, des laboratoires publics ou privés. 


\section{Robust Dynamic Hand Gesture Interaction using LTE Terminals}

\author{
Weiyan Chen \\ Key Laboratory of High Confidence \\ Software Technologies (MoE) \\ Department of Computer Science, \\ School of EECS, Peking University \\ Beijing, China \\ chenwy1117@pku.edu.cn \\ Rong Zheng \\ McMaster University \\ Harbin Institute of Technology \\ (Shenzhen) \\ Hamilton, Canada / Shenzhen, China \\ rzheng@mcmaster.ca
}

\author{
Kai Niu \\ Key Laboratory of High Confidence \\ Software Technologies (MoE) \\ Department of Computer Science, \\ School of EECS, Peking University \\ Beijing, China \\ xjtunk@pku.edu.cn \\ Dan Wu \\ Peking University \\ Beijing, China \\ dan@pku.edu.cn
}

\author{
Deng Zhao \\ China University of Geosciences \\ (Beijing) \\ Beijing, China \\ dengzhao.cugb@gmail.com
}

\author{
Wei Wang \\ Nanjing University \\ Nanjing, China \\ ww@nju.edu.cn
}

\author{
Leye Wang \\ Peking University \\ Beijing, China \\ leyewang@pku.edu.cn
}

\author{
Daqing Zhang* \\ Peking University \\ Telecom SudParis \\ Beijing, China / Evry, France \\ dqzhang@sei.pku.edu.cn
}

\begin{abstract}
Device-free hand gesture is one of the most natural ways to interact with everyday objects. However, existing WiFi-based gesture recognition solutions are typically restricted to indoor environments due to limited outdoor coverage. Furthermore, to achieve high sampling rates, they may interfere with normal data transmissions. In this paper, we aim to develop a robust dynamic gesture interaction system that can be ubiquitously deployed using Long-term Evolution (LTE) mobile terminals. Through both empirical studies and in-depth analysis using the Fresnel zone model, we reveal the key factors that contribute to the repeatability and discernibility of gestures. We show that the optimal location and orientation to perform gestures indeed exist and can be identified without prior knowledge of the position of LTE base stations (BSs) relative to a terminal. Guided by the design principles derived from Fresnel zone characteristics around a $4 \mathrm{G}$ terminal, we design highly repeatable and discernible gestures with salient received signal profiles. A gesture interaction system has been developed and implemented to achieve robust recognition with this careful design. Extensive experiments have been conducted in both indoor and outdoor environments, for different relative placements of mobile terminal and BS, and with different users. The proposed system can automatically identify the direction of BSs with a median error of less than 15 degrees and achieve gesture recognition accuracy as high as $98 \%$ in all scenarios without the need to acquire any training data.
\end{abstract}

\section{CCS CONCEPTS}

-Human-centered computing $\rightarrow$ Ubiquitous computing; Activity centered design; Gestural input; Ubiquitous and mobile computing systems and tools; • Information systems $\rightarrow$ Mobile information processing systems.

\section{KEYWORDS}

LTE signal, Gesture recognition, CSI ratio, Device-free sensing, Channel State Information

\section{INTRODUCTION}

Device-free hand gesture offers one of the most natural ways for Human-Computer Interaction (HCI). It allows users to interact with everyday objects and wearable devices of limited form factors in a non-intrusive manner. Hand gestures can be conceptually divided into static gestures and dynamic gestures. Dynamic hand gestures usually provide richer information than static gestures because of the incorporation of motion. Existing approaches to device-free hand gesture recognition mainly fall into four categories: visionbased [13, 23], acoustic-based [22, 28], Radio Frequency (RF) based using specialized devices such as mmWave and Ultra-wideband radars [17, 38] and WiFi-based solutions [2, 4, 15, 37, 40]. Among them, WiFi-based solutions utilize Channel State Information (CSI) extracted from WiFi signals received at a mobile terminal to recognize various hand gestures. Such solutions are attractive due to their low costs and no need for hardware modification. Despite successful experimental demonstrations in several application scenarios, WiFibased solutions are typically restricted to indoor environments due to limited outdoor coverage, and may interfere with normal data transmissions to acquire continuous, uniform and high sampling rates. Furthermore, there lacks a principled way to design RF-based 


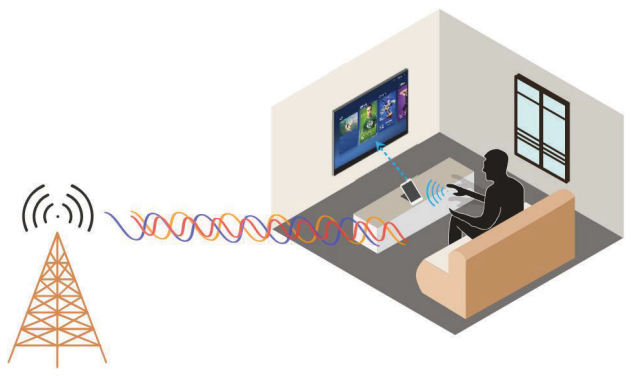

(a) Indoor scene

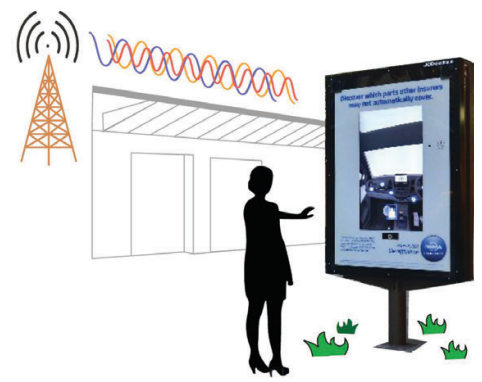

(b) Outdoor scene

Figure 1: Gesture interaction in indoor and outdoor scenes.

gesture interaction systems. Specifically, repeatability and discernibility are two key requirements for such systems. Repeatability means that features extracted from the same gesture performed by different people at different time, should have similar signal patterns. Discernibility, on the other hand, means that different gestures are distinguishable in the presence of minor motion variations, user diversity, environmental changes, etc. It is important to understand what factors contribute to the repeatability and discernibility of RF-based device-free gesture recognition, and how to design robust dynamic hand gestures accordingly.

In this work, we aim to design a robust dynamic gesture interaction system that can be ubiquitously deployed using $4 \mathrm{G}$ mobile terminals (Figure 1). For sensing modality, we choose Long-term Evolution (LTE) signals due to their wide-area coverage and high penetration rates of $4 \mathrm{G}$ LTE technologies in the past decade. Since the LTE BSs are widely distributed, no extra deployment is needed during gesture interaction. Only existing terminals (such as smartphones) need to be employed. LTE technologies have the additional advantage that mobile terminals can extract CSI directly from existing broadcast signals continuously transmitted from $4 \mathrm{G}$ BSs. Thus, no out-of-band transmissions are needed which may disrupt normal usages of wireless interfaces as in WiFi-based approaches. We further investigate the following questions: (1) Can we use LTE signals for dynamic hand gesture interaction? (2) What types of gestures are the most discernible for recognition? (3) Are LTE signal variation patterns location-dependent and orientation-dependent? If so, how to identify the best location and orientation that give the highest repeatability? In addition to answering these questions through empirical studies, we employ the Fresnel zone model [26, 29, 35], a model in propagation theory to quantify reflections and diffraction between a transmitter and a receiver, to unravel the relationship between how and where gestures are performed and their repeatability and discernibility.

Guided by the theoretical insights, we propose guidelines for gesture interactions that are not only applicable to LTE signals but can also be applied to other wireless systems that utilize the orthogonal frequency-division multiplexing (OFDM) modulation such as WiFi and 5G. We design several gestures for robust interactions using a $4 \mathrm{G}$ terminal and an LTE BS. We find that the performance of gesture recognition is highly dependent on the relative position between the gestures and the transceivers. Since the locations of LTE BSs are generally not known a priori for users, a simple procedure is devised to determine good near-terminal locations to perform the gestures with minimum human intervention. The main contributions of this work can be summarized as follows:

- Empirical studies have been conducted to demonstrate the feasibility of dynamic hand gesture interaction using LTE terminals and reveal that the variations of received signals are highly dependent on the locations and orientations of gestures performed as well as the gestures around a $4 \mathrm{G}$ terminal.

- By analyzing the Fresnel zones around a 4G terminal, we develop practical guidelines on where and what types of gestures should be performed to achieve the best repeatability and discernibility.

- Since the signal source is usually unknown in $4 \mathrm{G}$ scenarios, a novel mechanism is devised to infer the unknown direction of $4 \mathrm{G}$ BSs.

- Extensive experiments show that our proposed LTE-based gesture interaction system ${ }^{1}$ can achieve recognition accuracy as high as $98 \%$ in various environments for different users without any training.

The rest of the paper is organized as follows. In Section 2, we provide background knowledge of LTE-based device-free sensing. In Section 3, an empirical study is conducted to show the key factors that contribute to the repeatability and discernibility of gestures using LTE signals. In Section 4.3, the Fresnel zone model is introduced and used to guide robust hand gesture design. We present the proposed LTE-based hand gesture interaction system in Section 5 and evaluate it in Section 6. In Section 7, we survey the related works followed by a conclusion in Section 8 .

\section{SENSING FUNDAMENTALS}

In this section, we first give a brief introduction to LTE signal formats and show how to extract CSI from LTE frames. Next, we introduce CSI ratio as a robust measure to mitigate phase offsets and noise in CSI for LTE-based device-free sensing. 


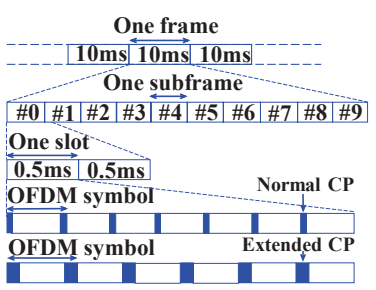

(a) Time Domain of LTE signals.

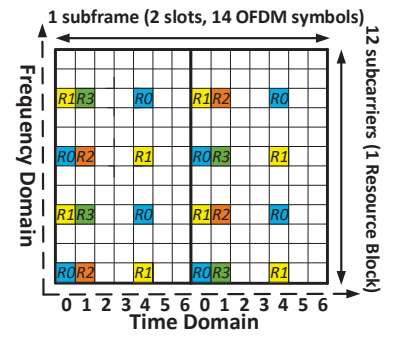

(b) Location layouts of CRSs in time-frequency resources of LTE signals.

Figure 2: The basic structure of LTE signals.

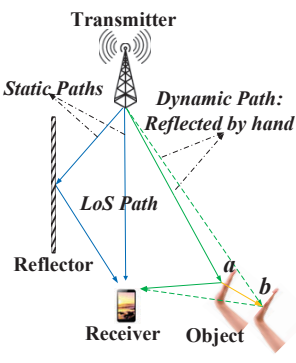

(a)

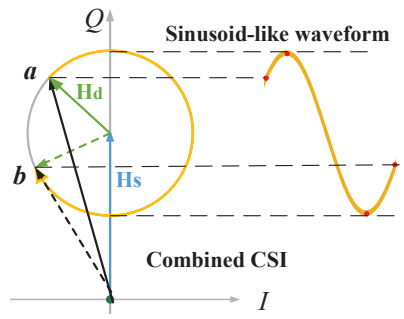

(b)

Figure 3: 4G scenarios and the CSI in a complex plane. (a) The LTE signal arrives at a receiving antenna along multiple paths. (b) CSI changes in a complex plane.

paths, given by

$$
\begin{aligned}
H(f, t) & =H_{S}(f, t)+H_{d}(f, t) \\
& =H_{S}(f, t)+\vec{a}(f, t) \cdot e^{-j 2 \pi \frac{d(t)}{\lambda}},
\end{aligned}
$$

consists of six (in the case of extended Cyclic Prefix (CP)) or seven OFDM symbols (in the case of normal $\mathrm{CP}$ ). In the frequency domain, an OFDM symbol occupies a series of subcarriers at a frequency interval of $\Delta f=15 \mathrm{kHz}$. Thus, the basic scheduling unit in LTE is called Resource Block (RB), which contains 12 subcarriers in frequency domain and lasts one slot $(0.5 \mathrm{~ms})$ in the time domain, as shown in Figure 2b. In each downlink RB (from a BS to a terminal), a Cell-Specific Reference Signal (CRS) with predefined symbols is transmitted by an LTE BS at four different locations with two CRSs separated by six subcarriers. For example, $R 0, R 1, R 2$ and $R 3$ in Figure $2 \mathrm{~b}$ are four common location layouts of CRSs in LTE systems. Therefore, a CRS forms a dense time-frequency grid at a fixed time and frequency intervals. It can be used to estimate channel state to improve the quality of communication.

\subsection{Extracting CSI from LTE Signals}

Channel state estimation is an effective means to improve system performance in wireless communication. Suppose that a BS transmits signal $X(f, t)$ on a given subcarrier $f$ at time $t$ in the frequency domain. The CSI $H(f, t)$ from the BS to a terminal can be estimated from the frequency-domain received signal $Y(f, t)$ as follows:

$$
H(f, t)=\frac{Y(f, t)}{X(f, t)}
$$

which is a complex-valued channel measurement. It represents how the environment around the transmitter and receiver (e.g., multipath effect, moving objects) affects the amplitude and phase of received LTE signals.

The LTE signal arriving at a receiving antenna along $N$ different paths can be grouped into two components: those along static paths and those along a dominant dynamic path [16, 26, 27], as shown in Figure 3a. The total CSI is the linear superposition of signals on all

\footnotetext{
${ }^{1} \mathrm{~A}$ live demo video is shown at https://youtu.be/e_OiQVkDExk.
}

where $H_{s}(f, t)$ is the static path component including signals through the Line-of-Sight (LoS) path and reflected paths from static objects, $\vec{a}(f, t)$ is the complex-valued representation of attenuation and initial phase offset of the dynamic path component $H_{d}(f, t)$. The latter varies with $d(t)$, the length of the reflected path induced by a moving object. $\lambda=c / f$ is the wavelength for the radio signal with frequency $f$, and $c$ is the speed of light. In the complex plane (Figure $3 \mathrm{~b}$ ), both $H_{s}(f, t)$ and $H_{d}(f, t)$ can be represented as vectors. $H_{s}(f, t)$ is a constant, while $H_{d}(f, t)$ varies with the dynamic path length $d(t) . e^{-j 2 \pi d(t) / \lambda}$ is the phase shift on the dynamic path. Whenever the movement of the object introduces one wavelength change in the dynamic path length, the corresponding phase changes $2 \pi$, and $H_{d}(f, t)$ rotates a cycle, generating a sinusoid-like waveform.

However, since the clocks on the BS and the terminal are generally not synchronized, the received CSI can be further characterized as:

$$
H(f, t)=\alpha_{\text {noise }}(f, t) e^{-j \cdot \theta_{\text {offset }}}\left(H_{s}(f, t)+H_{d}(f, t)\right),
$$

where $\alpha_{\text {noise }}$ denotes the noise in amplitude, $e^{-j \cdot \theta_{\text {offset }}}$ denotes the random phase offset in each CSI sample due to Carrier Frequency Offset (CFO), Sample Frequency Offset (SFO) [32], and so on. These phase offsets result in the randomness of phase information in CSI - a key challenge for sensing human activities using LTE signals.

\subsection{CSI Ratio}

To eliminate the randomness of phase offsets in CSI, we note that modern mobile devices like smartphones support MIMO technology [5]. Since the antennas in the same LTE adapter share the same clock, they have the same CFO, SFO and so on. Thus, we introduce the CSI ratio $[15,33]$ between two antennas as a new base signal for LTE-based sensing to suppress the impulse noise in amplitude and eliminate these phase random offsets in CSI of LTE signals. 


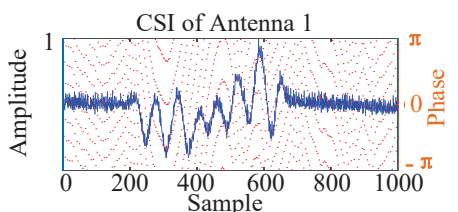

CSI of Antenna 2

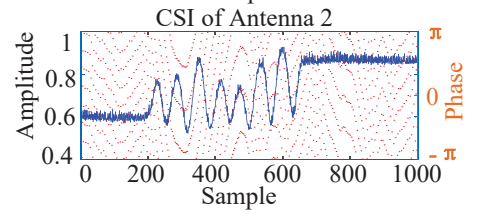

(a)
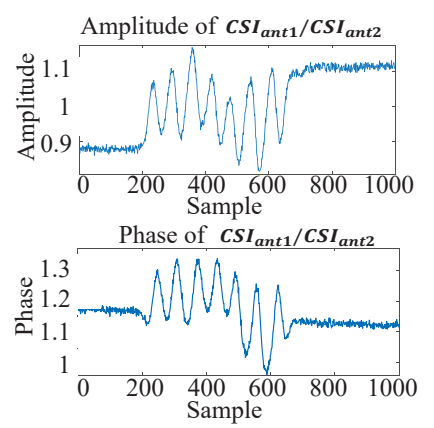

(b)

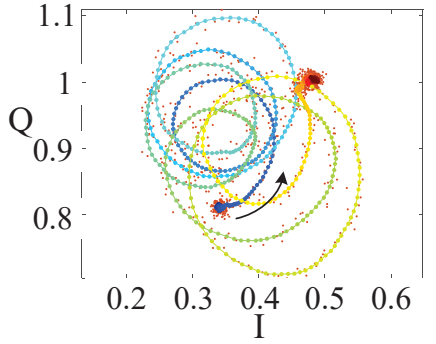

(c)

Figure 4: The impulse noise in CSI amplitude and random offset in CSI phase are removed in CSI ratio. (a) Amplitudes (blue lines) and phases (orange dots) of two antennas. (b) Amplitude and phase of CSI ratio. (c) The change of CSI ratios in complex plane (from the blue point to the red point).

The CSI ratio is defined as follows:

$$
H_{\text {ratio }}(f, t)=\frac{H_{s 1}(f, t)+\vec{a}_{1}(f, t) e^{-j 2 \pi \frac{d_{1}(t)}{\lambda}}}{H_{s 2}(f, t)+\vec{a}_{2}(f, t) e^{-j 2 \pi \frac{d_{1}(t)}{\lambda}} \cdot e^{-j 2 \pi \frac{\Delta d}{\lambda}}},
$$

where $H_{s 1}(f, t)$ and $H_{s 2}(f, t)$ are the static path components of the CSIs received by two different antennas in the same LTE adapter, respectively. While $\vec{a}_{1}(f, t)$ and $\vec{a}_{2}(f, t)$ are the attenuations and initial phase offsets of the antennas' dynamic path components. $\Delta d$ denotes the dynamic path length difference between the two antennas due to their different physical locations, which can be considered as a constant for a small-scale movement.

Figure 4 shows the raw CSI and CSI ratio of two antennas when an iron bucket moves away from the receiver along a straight line opposite to the transmitter. Comparing Figure 4a and Figure 4b, we find that the time-varying phase noise has been eliminated effectively and the high amplitude impulse and burst noise in raw CSI amplitude can be suppressed. Therefore, the CSI ratio between two antennas is a more robust base signal than the CSI of a single antenna. More importantly, we can observe that CSI ratio circles around approximately 7 times in the complex plane as the bucket moves $50 \mathrm{~cm}$ in Figure 4c. It has been proved in $[15,33]$ that the CSI ratio induced by a single moving (reflective) target have following properties:

- The CSI ratio mitigates both amplitude and phase noises of CSI effectively, and varies in a circular pattern in the complex plane.

- If the change in the reflection path length is exactly one wavelength, the CSI ratio will rotate exactly $2 \pi$ in the complex plane.

- As the reflection path length increases, the CSI ratio rotates clockwise, and counter-clockwise otherwise.

To estimate the change in the reflection path, we first calculate the phase change of tangent vectors of CSI ratio between two consecutive sample points in each subcarrier. The cumulative phase difference $\Delta p$ of tangent vectors indicates the overall phase change of dynamic component of CSI ratio induced by a target gesture movement. For more robust results, we average the phase differences

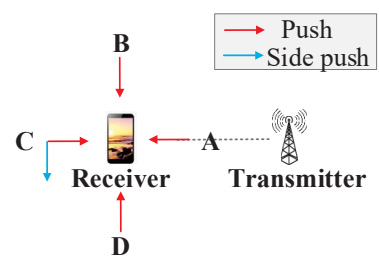

(a) Experiments for the same gesture.

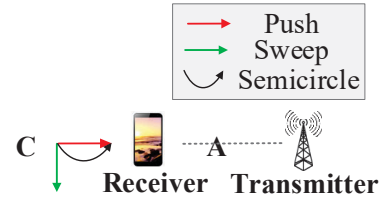

(b) Experiments for different gestures.
Figure 5: Testbed setup.

over all subcarriers. The resulting change in the reflection path length induced by the gesture can be thus calculated as $D=\frac{\lambda \Delta p}{2 \pi}$.

\section{EMPIRICAL STUDY ON CSI RATIOS INDUCED BY DYNAMIC HAND GESTURES}

In this section, we conduct empirical studies to investigate the repeatability and discernibility of LTE signal variations induced by hand gestures at different locations and orientations relative to a mobile terminal. Since CSI ratios are robust to noise and phase offsets, and can effectively capture channel variations due to a moving target, we use them to represent motion-induced CSI variations in subsequent discussion.

\subsection{Testbed Setup}

A small testbed is set up using an indoor femtocell as the transmitter and a USRP B210 platform as the terminal in the experiments. The transceivers are placed $5 \mathrm{~m}$ apart at $1 \mathrm{~m}$ height, and are equipped with one and two commonly used vertically polarized antennas, respectively. The transmitted signals cover $1.4 \mathrm{MHz}$ bandwidth centered on $2.165 \mathrm{GHz}$ band. CSI is captured at the rate of 100 samples per second at the receiver.

\subsection{Empirical Study}

First, we explore the repeatability of the same gesture around the terminal by performing the gesture at four locations $(A, B, C$ and $D)$ 


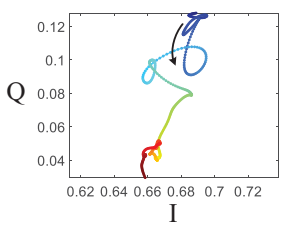

(a) Push at $A$

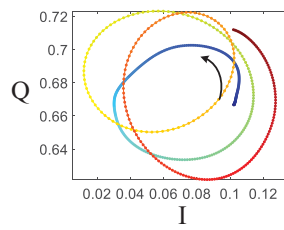

(b) Push at $B$

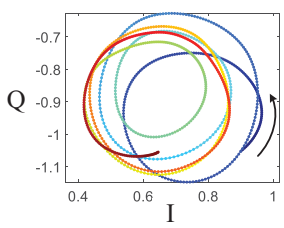

(c) Push at $C$

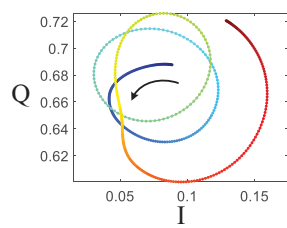

(d) Push at D

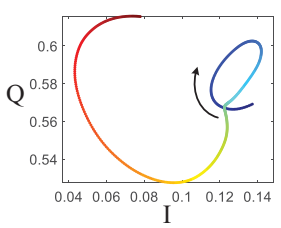

(e) Side push at $C$

Figure 6: CSI ratios in the complex plane induced by push movements at four different locations in $A, B, C$ and $D$.

Table 1: Mean values and variances of reflection path length changes of push gesture at four locations $(A, B, C$ and $D)$.

\begin{tabular}{ccccc}
\hline Location & $A$ & $B$ & $C$ & $D$ \\
\hline Mean value $(m)$ & 0.2209 & 0.4738 & 0.8032 & 0.4671 \\
Variance $\left(m^{2}\right)$ & 0.0149 & 0.0015 & 0.0012 & 0.0022 \\
\hline
\end{tabular}

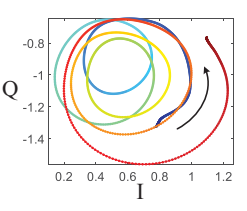

(a) Push at $C$

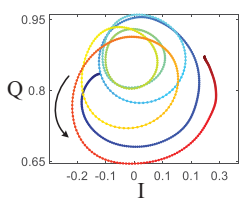

(b) Semicircle at $C$

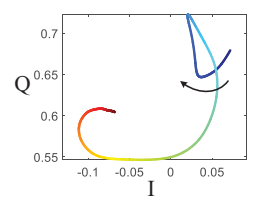

(c) Sweep at $C$
Figure 7: Discernibility among the received signal variations of different gestures at location $C$.

along a circle of radius $40 \mathrm{~cm}$ centered on the terminal (Figure 5a). Figure 6a-6d illustrate the changes of CSI ratios in a complex plane for the push gesture at four locations. In all figures, CSI ratios start from blue points and end at the red points. We can observe that with the exception of $A$, the CSI ratios mostly follow counterclockwise circles. This is consistent with the property that CSI ratios rotate counterclockwise when the reflection path length decreases as introduced in Section 2.3. However, there are fewer circles at $B$ and $D$ than those at $C$.

Next, we perform the same push gesture 30 times at each location, and calculate the mean values and variances of reflection path length changes induced by the movement. As shown in Table 1, the mean values and the variances at $A, B, C$ and $D$ are quite different. We observed that the repeatability of signal variations induced by the same gesture is location-dependent. Among all four locations experimented, gestures at $A$ and $C$ have the least and most repeatability, respectively.

To assess the impact of gesture orientations, we consider two orthogonal directions of a single push gesture, namely, push (red arrows) and side push (blue arrow) in Figure 5a. Figure 6c and 6e shows the CSI ratios of push and side push at location $C$ with $90^{\circ}$ of angle difference. Clearly, the CSI ratios exhibit very different patterns for the same gesture with different orientations. We observed that the received signal variations of the same gesture are orientation-dependent.
Lastly, we investigate the discernability of different gestures by comparing the CSI ratios of multiple gestures (Figure $5 \mathrm{~b}$ ) performed at the same location. In addition to push, we also perform two other gestures sweep and semicircle. Sweep is similar to side push except that the user's palm faces the receiver. A semicircle has identical starting and ending locations as push but follows a half circle instead of a straight line. All gestures are performed at $C$. Figure 7 shows that the CSI ratios of push and semicircle gestures have quite similar patterns and the same number of circles. In contrast, sweep generates a very different pattern. We thus observe that hand gestures of different spatial motions do not always generate distinctive signal variation patterns. Since some gestures are easier to be differentiated than others, special considerations are needed to design highly discernible gestures.

In summary, we observe empirically that i) the repeatability of signal variations of the same gesture are location-dependent and orientation-dependent, and ii) the signal variations induced by different hand gestures may not be discernible. Consistent observations have been made from experiments in outdoor environments using different types of commercial BSs (both microcell and macrocell).

\section{USING FRESNEL ZONE MODEL TO GUIDE ROBUST HAND GESTURE DESIGN}

In this section, we first introduce the Fresnel zone model to understand the relationship between received signal variations and hand movements. Then, based on the characteristics of Fresnel zones around a 4G terminal, we derive gesture design principles and present gesture design for robust hand gesture interaction.

\subsection{Fresnel Zone Model and Its Characteristics around A Receiver Terminal}

When transmitter $T x$ transmits RF signals with a wavelength of $\lambda$ to receiver $R x$ in free space, Fresnel zones refer to the concentric ellipses with foci of the transmitter and the receiver as illustrated in Figure $8[26,30,34,35]$. The boundary of the $n$th Fresnel zone is given by,

$$
\left|T x P_{n}\right|+\left|P_{n} R x\right|-|T x R x|=n \lambda / 2,
$$

where $P_{n}$ is a point on the $n$-th ellipse and $|\cdot|$ represents the Euclidean distance. The innermost ellipse $(n=1)$ is called the first Fresnel zone. The $n$-th Fresnel zone corresponds to the elliptical annuli between the $(n-1)$-th and $n$-th ellipses. Clearly, the width of the Fresnel zone keeps decreasing from the inside to the outside, approaching $\frac{\lambda}{2}$. 


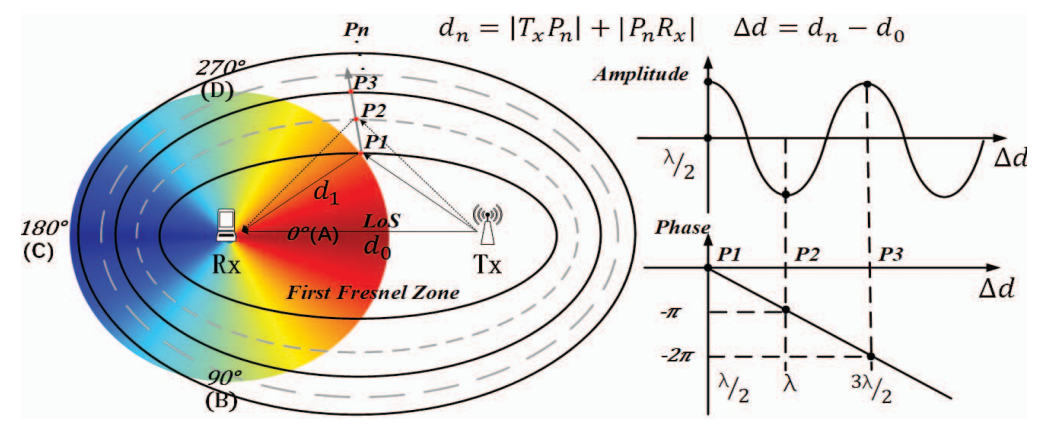

Figure 8: Geometry of the Fresnel zone and its distribution around a terminal.

Table 2: Design principles for robust gestures

\begin{tabular}{l|l|l}
\hline Property of Fresnel Zones & CSI Ratio Variation Pattern & Design Principles \\
\hline \hline $\begin{array}{l}\text { Diffraction effects in 1st Fresnel Zone (the } \\
\text { red area) }\end{array}$ & $\begin{array}{l}\text { Poor repeatability of CSI ratio signal varia- } \\
\text { tions }\end{array}$ & (I) Avoiding gesturing in red region \\
\hline $\begin{array}{l}\text { Evenly spaced Fresnel zones in the blue area } \\
\text { due to reflection effects }\end{array}$ & $\begin{array}{l}\text { Good repeatability of CSI ratio signal varia- } \\
\text { tions }\end{array}$ & (II) Gesturing in the blue area \\
\hline $\begin{array}{l}\text { Crossing two adjacent Fresnel zones corre- } \\
\text { sponds to a reflection path change of one } \\
\text { wavelength }\end{array}$ & $\begin{array}{l}\text { The dynamic component of CSI ratio moves } \\
\text { one cycle in the complex plane }\end{array}$ & $\begin{array}{l}\text { (III) Hand moving distance and patterns can } \\
\text { be easily profiled when the hand crosses mul- } \\
\text { tiple Fresnel zones perpendicularly }\end{array}$ \\
\hline
\end{tabular}

When a target is in the first Fresnel zone, diffraction is dominant $[35,36]$. The gain of the received signal is the sum of the energy bypassing the target, which depends on the size and position of the target. In contrast, reflection becomes dominant outside the first Fresnel zone. Under the assumption that there is only one dominant reflection path from a target, the received signal is the aggregation of the two signals along the direct path and reflected path. Since reflection introduces a phase shift of $\pi$, when the target is located in the boundaries of odd Fresnel zones (or even Fresnel zones), the two signals have the same phases (or destructive phases) but different amplitudes, leading to superposed stronger received signals (or weaker received signals). As illustrated in Figure 8 , when a hand continuously crosses outwards (or inwards) the boundaries of Fresnel zones, the amplitudes of the received signal exhibit a sinusoid pattern, while its phase increases (or decreases) with the reflection path length. Whenever the length of the reflection path changes by $\lambda$, the phase of dynamic vector changes by $2 \pi$. By the definition of CSI ratio in Section 2.3, $\lambda$ reflection path length change results in $2 \pi$ phase change in the dynamic component, or equivalently rotation of a complete circle. Furthermore, when the reflection path increases, the dynamic component rotates clockwise, and counter-clockwise vice versa. The exact pattern of the received signal variations induced by a hand gesture depends on the number and direction of the Fresnel zone crossed.

\subsection{Implication on Gesture Design}

As shown in Figure 8, the Fresnel zones around a terminal $(R x)$ are unevenly distributed. Such an uneven distribution has a number of important implications on gesture design. First, in the red area centered around the first Fresnel zone, where diffraction dominates when a hand moves in that area, any slight deviation of a hand moving trajectory leads to a significant difference in signal variation patterns at the receiver. In contrast, as observed in Section 3.2, in the high-density Fresnel zone area (the blue area in Figure 8), similar hand moving trajectories crossing the Fresnel zones lead to similar signal variation patterns at the receiver due to the even spacing of Fresnel zones there. Second, in the dense Fresnel zones, given the same hand moving distance, when the hand moves perpendicular to the boundaries of Fresnel zones, more zones can be crossed. Equivalently, there are more phase changes in CSI or CSI ratio. Third, as observed in Section 3.2, when performing the same gesture at the same location but different orientations, it is expected that the moving trajectories cross different numbers of Fresnel zones. As a result, they induce different signal variations. Lastly, to distinguish different gestures, we would like to have repeatable signal variation patterns for individual gestures, but very different signal variation patterns among different gestures, e.g., crossing different numbers of Fresnel zones or in different directions.

To this end, we summarize the key properties of the Fresnel zones around a $4 \mathrm{G}$ terminal and the corresponding guiding principles for robust gesture design in Table 2.

\subsection{Robust Hand Gestures}

From the design principles I and II, we know that for better repeatability, gestures should be performed in the blue area (called the optimal area). Principle III implies that gesture movements should be perpendicular to the Fresnel zone boundaries (called the optimal orientation) and cross the Fresnel zone continuously. What remains to tackle is to design multiple discernible gestures. We propose the template of gesture interaction which can be used in both indoor and outdoor environments. 
Table 3: Template for gesture interaction.

\begin{tabular}{l|l|l}
\hline Pattern & Movement & Example \\
\hline$+\mathrm{d}$ & Away from the receiver in straight line & Forward \\
\hline$-\mathrm{d}$ & Towards the receiver in straight line & Backward \\
\hline$+\mathrm{d}-\mathrm{d}$ & $\begin{array}{l}\text { Away from and towards the receiver in } \\
\text { straight line }\end{array}$ & Continue \\
\hline$-\mathrm{d}+\mathrm{d}$ & $\begin{array}{l}\text { Towards and away from the receiver in } \\
\text { straight line }\end{array}$ & Cancel \\
\hline$+\mathrm{d}-\mathrm{d}+\mathrm{d}-\mathrm{d}$ & $\begin{array}{l}\text { Away from and towards the receiver twice } \\
\text { in circle line }\end{array}$ & Switch on \\
\hline$-\mathrm{d}+\mathrm{d}-\mathrm{d}+\mathrm{d}$ & $\begin{array}{l}\text { Towards and away from the receiver twice } \\
\text { in circle line }\end{array}$ & Switch off \\
\hline
\end{tabular}

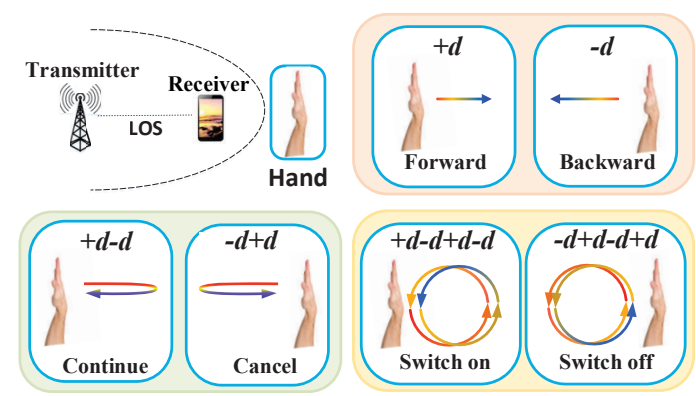

Figure 9: Example gestures for interaction. Gestures start from the ends (in red) and end at the heads (in blue) of arrows with the hand palm facing to the terminal.

To eliminate the need for acquiring training data and to achieve lightweight and robust gesture recognition, we only consider one salient pattern in rotation directions of CSI ratios, namely, clockwise and counter-clock rotations. As a hand crosses a series of Fresnel zones, the reflection path length changes continuously. Denote the increase of reflection path length by symbol + , and the decrease by symbol - . When the reflection path increases by a distance $d$ (e.g., when the hand moves away from the receiver), the corresponding signal variation pattern is denoted by $+d$. Similarly, one can define other unique signal variation patterns such as $(-d),(+d-d),(+d-$ $d+d)$, etc. We show six basic signal variation patterns in Table 3 . Noted that more gestures can be designed by combining different directions and distances.

By mapping well-defined signal variation patterns to different gestures, we can achieve gesture recognition with high accuracy without training. In Figure 9, six gestures are shown as examples for remote device control such as TVs in indoor scenarios and digital screens in outdoor scenarios. These gestures can be used to switch on and switch off a device, continue and cancel an action, move forward and backward, etc.

\section{LTE-BASED HAND GESTURE RECOGNITION}

As shown in Figure 10, the proposed gesture interaction system consists of five main modules: data acquisition, profile extraction, transmitter's direction estimation, gesture recognition, and device control. We acquire the data from downlink LTE signals, which can

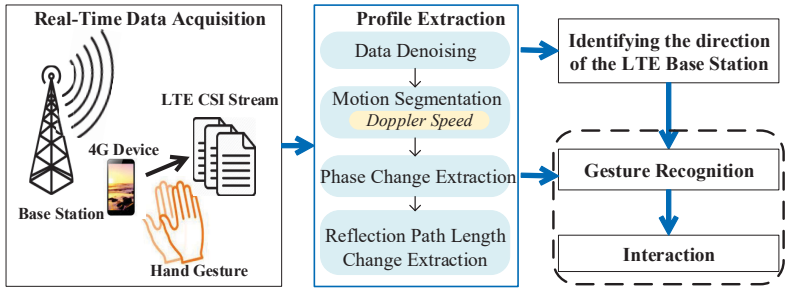

Figure 10: Flow chart of LTE-based gesture interaction.

be used to extract the path length change profile. Our system estimates the direction of the BS and recognizes gestures sequentially with this extracted profile as input.

\subsection{Data Acquisition}

A 4G terminal with two antennas extracts CSI from downlink LTE signals transmitted from a BS (either indoor or outdoor). Two CSI streams from the receiver are collected and are used to derive the CSI ratio for each subcarrier as defined in Equation (4). There are a total of 12 subcarriers utilized out of a maximum 200 subcarriers in LTE. The resulting CSI ratios are then further processed by MATLAB to extract motion profiles.

\subsection{Profile Extraction}

5.2.1 Denoising. To eliminate the remaining noises, we apply a Savitzky-Golay filter to the CSI ratios, which smooths digital samples without introducing excessive distortion by fitting successive subset of data points with low degree polynomials. In our implementation, the polynomial order and the frame length are set as 2 and 11 , respectively.

5.2.2 Motion segmentation. We identify data segments corresponding to the designed hand movements by observing the Doppler speed of the CSI ratio inputs. We apply the MUSIC algorithm [12] to extract Doppler speed induced by a movement as shown in Figure 11a. The basic idea of MUSIC is to analyze the eigenvectors of the $M \times M$ correlation matrix $R_{x}$ of the received $M$ CSI samples:

$$
R_{x}=E\left[Z(f) Z(f)^{*}\right],
$$

where $*$ refers to the hermitian (conjugate transpose) of the vector and $Z(f)$ denotes the continuous $M$ CSI ratios samples obtained from the subcarrier with frequency $f$. MUSIC partitions the eigenvectors of $R_{x}$ into 2 subspaces: the signal subspace $E_{S}$ constructed by $L$ eigenvectors and the noise space $E_{n}$ constructed by eigenvectors corresponding to the smallest $M-L$ eigenvalues. These two subspaces are orthogonal. Thus, the speed spectrum function can be expressed as:

$$
P(v)_{M U S I C}=\frac{1}{\vec{a}(v)^{*} E_{n} E_{n}{ }^{*} \vec{a}(v)},
$$

where $v$ denotes the path length change speed, $\vec{a}(v)$ equals to $\left[1, \mathrm{e}^{-\mathrm{j} 2 \pi \mathrm{f} \frac{\mathrm{v} \Delta t_{2}}{\mathrm{c}}}, \mathrm{e}^{-\mathrm{j} 2 \pi \mathrm{f} \frac{\mathrm{v} \Delta \mathrm{t}_{3}}{\mathrm{c}}}, \ldots, \mathrm{e}^{-\mathrm{j} 2 \pi \mathrm{f} \frac{\mathrm{v} \Delta \mathrm{t}_{\mathrm{M}}}{\mathrm{c}}}\right]$ and $\Delta t_{i}$ denotes the sampling interval between these two samples. We use the sliding window method to process the data. For a good trade-off between processing time and profile resolution, the window size is set to 50 samples with a step size of 10 . If the profile power is above a 


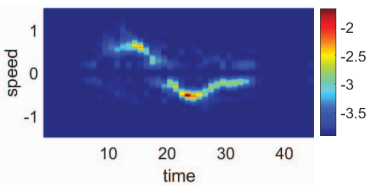

(a) Motion segmentation

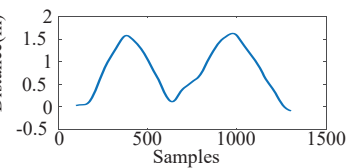

(a) Start from $0^{\circ}(\mathrm{LoS})$.

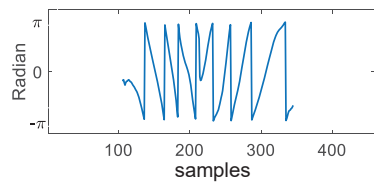

(b) Tangent phase

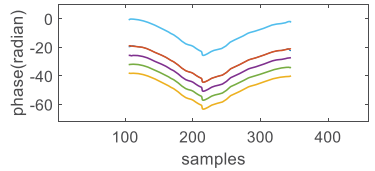

(c) Unwrapped tangent phases (6 subcarriers)

Figure 11: Process of profile extraction.

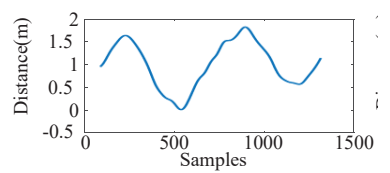

(b) Start from $90^{\circ}$.

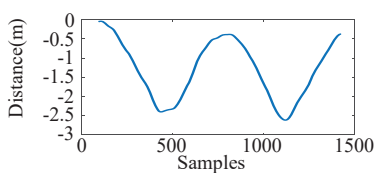

(c) Start from $180^{\circ}$.

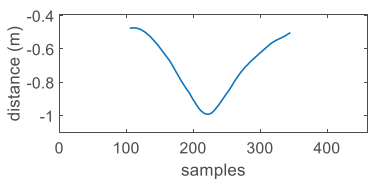

(d) Change of reflection path length

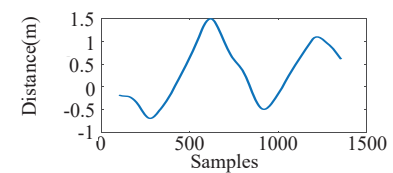

(d) Start from $270^{\circ}$.

Figure 12: Reflection path length change when walking around a terminal in two circles clockwise from four different starting locations.

certain threshold, motion is detected. The threshold is pre-defined but can be updated based on known periods of inaction.

5.2.3 Phase change Extraction. Recall that the phase changes of the dynamic component of the CSI ratio have quantitative relationships with dynamic path length changes induced by hand movements. As mentioned in Section 2.3, once motion segments are identified, we calculate the vector along the tangential direction by subtracting a complex value of each sample point from its immediate successor. The phase changes of the resulting sequence (Figure 11b) give the rotation direction of the CSI ratio samples in the complex plane (i.e., the moving direction of the circular arcs) and the changes in the reflection path length. After that, we unwrap the phase sequence in each subcarrier by adding multiples of $2 \pi$ when the phase changes from $-\pi$ to $\pi$ (Figure 11c).

5.2.4 Determining the Reflection Path Length Change. In Section 2.3, we know that a change in the reflection path by one wavelength induces a phase change of $2 \pi$ in the complex plane. We first take the average of the unwrapped phase changes among all subcarriers and calculate the path length changes by multiplying phase changes with $\frac{\lambda}{2 \pi}$, where $\lambda$ denotes the wavelength. We then apply a Savitzky-Golay filter to smooth the result. As shown in Figure 11d, the average reflection path changes over time constitute the profile of a gesture, which is used in direction estimation and gesture recognition.

\subsection{Identifying the Direction of the BS}

Determining the best location and orientation of dynamic hand gestures require the knowledge of the direction of a $4 \mathrm{G}$ BS (transmitter) relative to the terminal, which is generally not known a priori for users.

The key idea is to leverage the uneven distribution of the Fresnel zones around the receiver to estimate the direction of the LTE BS. As shown in Figure 8, the Fresnel zones are sparse near the LoS (the red colored area) and are dense at the opposite direction of the BS (the blue colored area). Therefore, when a user walks around the receiver in circles at a constant speed, we will observe different reflection path profiles due to the changes in Fresnel zone density.

Figure 12 shows the reflection path change when walking along two circles with different starting locations. We can see that all of them undergo two valleys and two peaks, corresponding to two walking circles. The peaks occur when the user is at the far end of the receiver (at $180^{\circ}$ in Figure 8), while the valleys occur when the user is between the transmitter and the receiver (at $0^{\circ}$ ).

Let $\Delta T$ be the time it takes from an arbitrary starting location to the first appearance of a peak in dynamic path changes. The amount of time to walk a single circle is given by $T=\left(t_{e}-t_{s}\right) /$ num_circles, where $t_{s}$ and $t_{e}$ are the start and finish times to complete all circles, and num_circles is the number of circles traversed by the user. The ratio between $\Delta T$ and $T$ is proportional to the angle from the starting point to the direction of $180^{\circ}$ (i.e., the opposite direction of the LTE BS). Specifically, the angle is calculated as: $\theta=\Delta T / T \times 360^{\circ}$.

\subsection{Gesture Recognition and Device Control}

To distinguish different gestures, we slice each path length change profile into monotone segments and assign each with a binary code. We denote the decrease and increase in reflection path length by binary codes ' 0 ' and ' 1 ', respectively. For instance, the profile in Figure $11 \mathrm{~d}$ is denoted as binary codes ' 01 '. Since the pre-defined gestures can also be represented as binary codes according to their signal variation patterns, by matching the binary codes of profiles and that of designed gestures, we can accurately recognize the performed gestures. To reduce false alarms, we only consider gestures that last less than 3 seconds with individual segments corresponding to path length changes less than 0.8 meters. Such assumptions are reasonable due to the limited range of motion of human limbs. The durations of any two segments should be no more than 1.5 times of one another.

Once a gesture is recognized, the system will send the corresponding control command to the device to be controlled. 


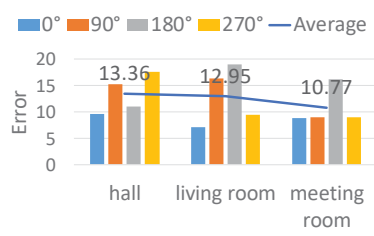

(a) Overall performance.

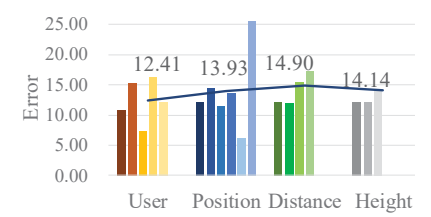

(b) Different impact factors.
Figure 13: Direction estimation errors of BS direction finding.

\section{EVALUATION}

In this section, we present the evaluation results of identifying the direction of a BS and gesture recognition under various circumstances.

\subsection{Identifying the Direction of the BS}

To obtain the ground truth location of the transmitter for direction estimation, we use an indoor femtocell as the transmitter. The hardware setup is similar to that in Section 3. The height of the receiver is $1 \mathrm{~m}$. Experiments are conducted in three indoor areas: a hall (of size $6 \times 9 \mathrm{~m}^{2}$ ), a living room (of size $7 \times 4 \mathrm{~m}^{2}$ ) with several furniture, and a large meeting room (of size $7 \times 6 \mathrm{~m}^{2}$ ) equipped with desks and chairs.

To verify the effectiveness and robustness of the proposed directionfinding method, we vary the user who conducts the experiments, the height of the BS, and the distance and the relative position between the transmitter and the receiver. In each experiment, we ask volunteers to circle around the receiver twice from four different starting locations in $0^{\circ}, 90^{\circ}, 180^{\circ}$ and $270^{\circ}$ (shown in Figure 8). Each experiment is repeated twenty times.

6.1.1 Overall performance. Figure 13a shows the overall performance in three test areas. The mean absolute errors (MAE) in three areas are $13.36^{\circ}, 12.95^{\circ}$ and $10.77^{\circ}$, respectively. We observe that the change of environments or starting location has little effect on the accuracy of direction finding. Note that the errors in all cases are under $15^{\circ}$. This implies that if we perform gestures at the optimal location $180^{\circ}$, the actual location falls into the sector ranging from $165^{\circ}$ to $195^{\circ}$, i.e., the dense blue area in Figure 8.

6.1.2 Impact of user diversity. We conduct experiments in the hall with the help of 5 volunteers ( 1 female and 4 males). The height of the transmitter is $2 \mathrm{~m}$ and the distance between the transmitter and the receiver is $4 \mathrm{~m}$. Figure 13b shows the estimated results for all 5 users. Though variation exists, all volunteers can reliably find the direction of the BS.

6.1.3 Impact of the relative position of the transmitter and the receiver. To explore the impact of relative positions of the transmitter and receiver, we put the transmitter at the center of the hall at height $2 \mathrm{~m}$ and place thereceiver at 6 locations around the transmitter with a space of $60^{\circ}$. The distance between the transmitter and the receiver is $3 \mathrm{~m}$. The results are shown in Figure 13b. It can be observed that the proposed direction finding method is generally robust to the relative positions. At position 6, the estimation error is larger than those at other positions. This is because when the receiver is close to a wall, the secondary reflection from the wall may be comparable to the primary reflection from the human body, causing deviation in the estimation result.

6.1.4 Impact of the distance between the transmitter and the receiver and the height of the BS.. In the next set of experiments, we vary the distance between the transmitter and the receiver $(3 \mathrm{~m}, 4 \mathrm{~m}$, $5 \mathrm{~m}$ and $6 \mathrm{~m})$, and the height of the transmitter $(1.5 \mathrm{~m}, 2 \mathrm{~m}$ and $25 \mathrm{~m})$. As shown in Figure 13b, the direction estimation error is consistently small in all settings.

\subsection{Gesture Recognition}

To evaluate the performance of gesture recognition, we conduct extensive experiments in three representative $4 \mathrm{G}$ scenarios, namely, both transceivers are indoor (Scenario 1), the transmitter is outdoor and the receiver is indoor (Scenario 2), and both transceivers are outdoor (Scenario 3).

Figure 14 shows the experimental settings for gesture recognition. Both indoor and outdoor commercial microcell BS are used as transmitters. A total of 16 receiver locations ( 9 indoor locations marked as red stars and 7 outdoor locations marked as blue stars) are evaluated in the test area. 10 volunteers ( 4 females and 6 males between age 19 to 27 with different heights and weights) have been recruited. We choose 6 gestures in Table 3 and denote them by letter $a$ to letter $f$ (top to bottom). All gestures are performed at the optimal location as inferred by the proposed direction finding method. At each location, each gesture is performed 30 times by each volunteer. The recognition accuracy is calculated by: (Total $-F P-F N) /$ Total, where Total, FP and FN denotes total number of trials, false positive and false negative of each gesture, respectively.

6.2.1 Scenario 1: Both transceivers are indoor. We consider 6 different receiver locations in 4 different environments as shown in Figure 14a. Figure 15a shows the average accuracy of recognizing different gestures. It can be observed that the average accuracy is higher than $95 \%$ in all locations. Minor degradation can be observed when gestures are performed in bedroom $2(R \times 4)$. This can be primarily attributed to the higher RF attenuation through two walls. We can also find that, although in the same rooms, $R \times 1$ and $R \times 5$ have lower accuracy than those of $R \times 2$ and $R \times 6$. This is because $R x 1$ and $R x 5$ are near walls. The secondary reflection from the wall interferes with the signal reflected by hand. As a general rule of thumb, we recommend that gestures should be performed away from objects in the environment.

6.2.2 Scenario 2: Transmitter is outdoor and receiver is indoor. We place a receiver at 3 different indoor positions for testing, all of which are roughly 100 meters away from an outdoor BS. As shown in Figure 14b, $R x 7$ is in the corridor of the building and behind the concrete wall of the meeting room where $R x 8$ and $R x 9$ are placed. Figure 15b shows the average accuracy of recognizing 6 gestures in 3 different indoor positions. We observed that $R x 7$ in the narrow corridor has lower recognition accuracy than other positions. This is due to the RF attenuation through the concrete wall, resulting in weak signal reflected by hand movements. 


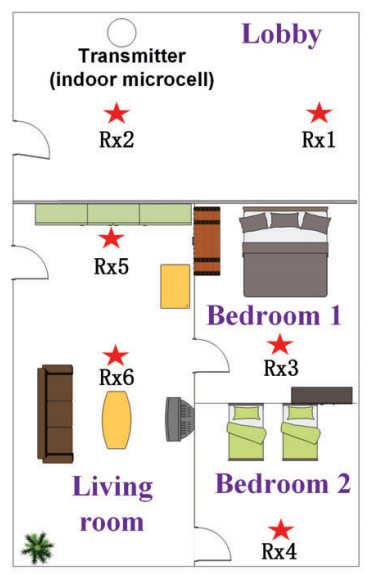

(a) Scenario 1 .

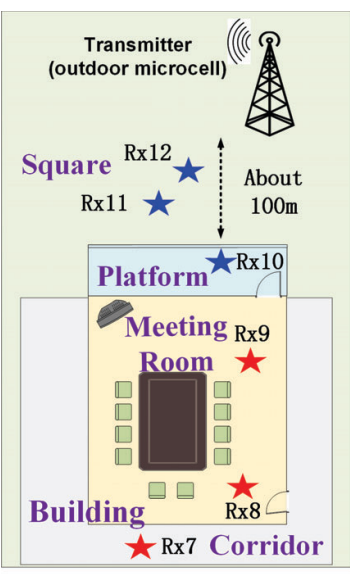

(b) Scenario 2 and 3(a).

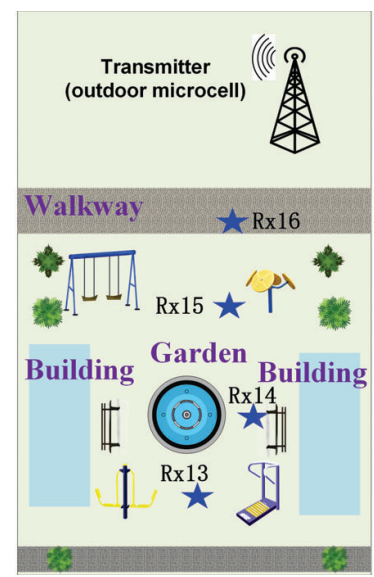

(c) Scenario 3(b).

Figure 14: Experimental settings for gesture recognition using commercially deployed indoor and outdoor microcell BSs.

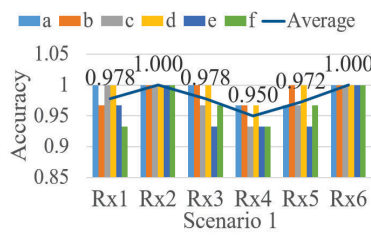

(a) Scenario 1 .

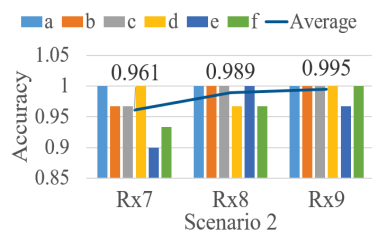

(b) Scenario 2 .

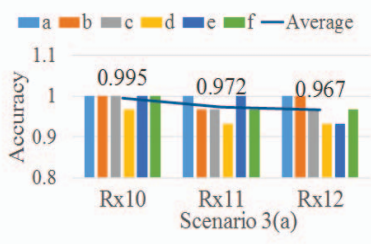

(c) Scenario 3(a).

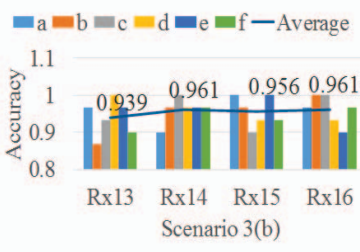

(d) Scenario 3(b).

Figure 15: Gesture recognition using an indoor and an outdoor microcell BS.

6.2.3 Scenario 3: Both transceivers are outdoor. To evaluate the performance of gesture recognition when the $4 \mathrm{G}$ terminal in outdoor environments, we conduct experiments in 7 locations of two typical outdoor environments (shown in Figure 14b and Figure 15c). In the first setting, $R x 10$ is on an open platform of the fifth floor of a building. $R x 11$ and $R x 12$ are on the ground open space between the building and a BS. In the second setting, $R \times 13, R \times 14$ and $R \times 15$ are in a garden of a residential area. $R \times 16$ is on the walkway between an outdoor BS and the garden. Figure $15 \mathrm{c}$ shows the recognition accuracy of the 6 gestures. We observed that the average recognition accuracy of $R x 10$ is higher than other locations in the environment shown in Figure 14b. This is a clear direct light-of-sight path between the BS and $R x 10$. We also observe from Figure $15 \mathrm{~d}$ that the average recognition accuracy of locations in Figure 14c is generally lower than that in Figure 14b. This can be attributed to complex multi-path propagation from trees, utility poles or pedestrians. Dynamic disturbances (e.g., from walking pedestrians) near the $4 \mathrm{G}$ terminal can introduce additional dynamic components beyond that from the designed hand gestures, inducing unexpected received signal variation patterns.

6.2.4 Impact of gesture locations. To further verify the impact of gesture locations, we compare the performance of gesture recognition in the optimal location and in the first Fresnel zone when the receiver locates in $R x 6$ of Figure 14a. Each gesture is performed

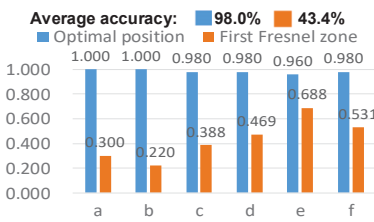

(a) Impact of gesture locations.

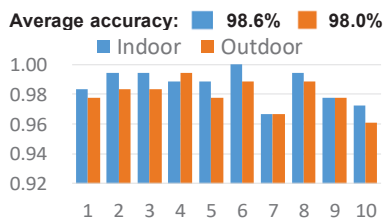

(b) Impact of user diversity.
Figure 16: Effects of gesture locations and user diversity.

60 times at the optimal position and in the first Fresnel zone. As shown in Figure 16a, the overall gesture recognition in the optimal area achieves accuracy as high as $98 \%$ which is significantly better than that in the first Fresnel zone. This confirms design principles I and II in Section 4.2 .

6.2.5 Impact of user diversity. We also evaluate the impact of user diversity on gesture recognition in both indoor and outdoor environments. We ask 10 volunteers to perform gestures at $R x 6$ in Figure 14a and $R x 4$ in Figure 14b. Each gesture is performed 30 times. Figure 16b shows that the performances of different users are marginally different in both indoor and outdoor environments, which indicates that the proposed system is robust to different users. The overall accuracy in indoor and outdoor are $98.6 \%$ and 
$98.0 \%$, respectively. Since the indoor location is closer to the BS and experiences less interference, the recognition accuracy at the locations is slightly higher than that of the outdoor locations.

In addition to the above experiments, we also tested the proposed system to control a TV remotely for one day in a smart home with the indoor microcell BS (see https://youtu.be/e_OiQVkDExk). Similar accuracy has been observed throughout the day.

\subsection{Discussion}

6.3.1 Limitations. Since the model assumes a single dynamic component, the recognition accuracy of the system decreases when there are multiple moving objects near the terminal. False negatives and false positives indeed exist in practice. A false negative (or miss reporting) happens when the execution of a gesture significantly deviates from the specification. For instance, when the range of motion is much smaller than $d$ or the actual orientation of the gesture is far from the optimal one, the resulting patterns may be dissimilar to the known profiles. A false positive (or false alarm) may occur in the presence of movements of the face, hand or other parts of the human body, such as raising hands, moving the body forth and back, or other moving objects in the surroundings. These null movements may generate patterns similar to the designed patterns Such errors can be mitigated by considering the duration of the gestures.

6.3.2 Applicability to other wireless systems. The proposed CSI ratio calculation and gesture recognition solution are applicable to other wireless systems as long as OFDM and MIMO are utilized in the physical layer. Examples include WiFi 802.11g and above, 5G cellular networks. The design principles derived from Fresnel zone analysis are expected to provide insights to the design of other RF sensing solutions.

\section{RELATED WORK}

\subsection{Non-RF based gesture recognition.}

Non-RF based techniques have been widely used in gesture recognition, including wearable devices $[9,31]$, computer vision $[13,18]$ and sound waves [14, 22, 28]. Wearable device based methods use dedicated sensors worn by users' hands for gesture recognition. For example, FDSVM [31] uses a wearable 3-dimensional accelerometer to recognize 12 gestures with high accuracy. Wearable ring-type [9] or watch-type [25] sensors have been used for recognizing various finger or hand gestures for text input or appliances control. However, these methods all require users to wear physical sensors, which is inconvenient. Computer vision based systems (such as Microsoft Kinectand Leap motion [13]) use cameras or infrared sensors to reconstruct the depth information from images. Although these approaches do not require a user to wear any sensors, these still rely on dedicated hardware and cannot work well in poor lighting conditions. Acoustic based approaches use speakers and microphones to gather echoes of the hand to recognize gestures [22]. LLAP [28] uses phase change of the sound signal for motion sensing. FingerIO [14] uses OFDM modulated sound signals for centimeter-level finger tracking. While promising, the sonar based systems only have limited sensing range and have risk of privacy leak due to the fact that devices record sound waves while working.

\subsection{RF-based gesture recognition.}

Recently, RF-based gesture recognition has been actively explored. For instance, SideSwipe [39] and AllSee [10] use TV and cellular transmissions to recognize hand, arm, and leg gestures, respectively. WiTrack [3] also uses custom radar transmissions to detect pointing gestures. Gestures can also be sensed with the Channel State Information (CSI) in an RF communication system like WiFi. WiKey [4] and Mudra [37] extract unique patterns in the time-series of CSI values of WiFi signals to identify different finger gestures. More recently, WiMu [24] applies the Short Time Fourier Transform (STFT) on denoised CSI stream and generates virtual samples to enable multi-user gesture recognition. Most of them are learningbased approaches. They rely on distinguishable feature patterns and use machine learning methods to classify different gestures. However, trained classifiers may not work properly when the system setting changes. Besides, these works fail to consider that the variations of received CSI signals are highly dependent on the locations and orientations of gestures. To overcome these problems, Widar 3.0 [40] derives and estimates the velocity profiles of gestures at the signal level and attempts to construct a consistent signal profile independent of location and orientation. However, it requires one transmitter and multiple receivers placed orthogonally around the users to record gestures from different views, which is usually not possible in $4 \mathrm{G} / 5 \mathrm{G}$ scenes.

\subsection{G/5G-based sensing.}

Benefiting from the wide radio signal coverage of $4 \mathrm{G}$ and $5 \mathrm{G}$ signals, it is attractive to leverage these ubiquitous signals for sensing [6]. With the CSI extracted from CRS of LTE signals, it is possible to localize the device with an error of $43 \mathrm{~m}$ Root Mean Square (RMS) for outdoor scenes [11] and with an error of $1 \mathrm{~m}$ by using fingerprinting-based approaches for indoor scenarios [19, 20]. System in [7] investigates the possibility to use LTE signals for crowd density estimation by extracting statistical features of Reference Signal Received Power (RSRP) in LTE. Works in [21] show the capability of LTE signals for motion detection. Besides 4G-based sensing, a few $5 \mathrm{G}$ prototype systems are proposed for preliminary human sensing, such as crowd-size detection [8] and walking speed recognition [8]. However, no gesture recognition systems using 4G/5G signals are reported before. In fact, existing studies on 4G/5G-based sensing mainly focus on coarse-grained activity, and there is no work exploring small scale movements like hand gestures.

\section{CONCLUSION}

In this paper, we empirically found that the repeatability and discernibility of gestures in LTE-based gesture recognition systems are location-dependent and orientation-dependent. By analyzing the Fresnel zone characteristics around a $4 \mathrm{G}$ terminal, we provided guiding principles for training-free robust gesture designs and developed a robust gesture interaction system. Extensive experiments showed that the proposed system can achieve high accuracy in estimating the direction of BSs and gesture recognition in various environments and configurations for different users. 


\section{ACKNOWLEDGEMENTS}

This research is supported by National Key Research and Development Plan under Grant No.2016YFB1001200, EU CHIST-ERA RadioSense Project, and Peking University Information Technology Institute (Tianjin Binhai).

\section{REFERENCES}

[1] 2019. LTE; Evolved Universal Terrestrial Radio Access (E-UTRA); Physical channels and modulation (3GPP TS 36.211 version 15.6.0 Release 15)

[2] Heba Abdelnasser, Khaled Harras, and Moustafa Youssef. 2018. A Ubiquitous WiFi-based Fine-Grained Gesture Recognition System. IEEE Transactions on Mobile Computing (2018), 1-1.

[3] Fadel Adib, Zach Kabelac, Dina Katabi, and Robert C. Miller. 2014. 3D Tracking via Body Radio Reflections. In 11th USENIX Symposium on Networked Systems Design and Implementation (NSDI 14). Seattle, WA, 317-329.

[4] Kamran Ali, Alex X. Liu, Wei Wang, and Muhammad Shahzad. 2015. Keystroke Recognition Using WiFi Signals. In Proceedings of the 21st Annual International Conference on Mobile Computing and Networking (Paris, France) (MobiCom '15). 90-102.

[5] Imee Ristika Rahmi Barani and Kin-Lu Wong. 2018. Integrated Inverted-F and Open-Slot Antennas in the Metal-Framed Smartphone for $2 \times 2$ LTE LB and $4 \times 4$ LTE M/HB MIMO Operations. IEEE Transactions on Antennas and Propagation 66, 10 (2018), 5004-5012.

[6] Weiyan Chen, Kai Niu, Dan Wu, Deng Zhao, Leye Wang, and Daqing Zhang. 2019. A contactless gesture interaction system using LTE (4G) signals. In Adjunct Proceedings of the 2019 ACM International foint Conference on Pervasive and Ubiquitous Computing and Proceedings of the 2019 ACM International Symposium on Wearable Computers. 260-263.

[7] Simone Di Domenico, Mauro De Sanctis, Ernestina Cianca, Paolo Colucci, and Giuseppe Bianchi. 2017. LTE-based passive device-free crowd density estimation. In 2017 IEEE International Conference on Communications (ICC). 1-6.

[8] Bahareh Gholampooryazdi, Isha Singh, and Stephan Sigg. 2017. 5G Ubiquitous Sensing: Passive Environmental Perception in Cellular Systems. In 2017 IEEE 86th Vehicular Technology Conference (VTC-Fall). 1-6.

[9] Lei Jing, Yinghui Zhou, Zixue Cheng, and Tongjun Huang. 2012. Magic Ring: A Finger-Worn Device for Multiple Appliances Control Using Static Finger Gestures. Sensors 12, 5 (2012), 5775-5790.

[10] Bryce Kellogg, Vamsi Talla, and Shyamnath Gollakota. 2014. Bringing Gesture Recognition to All Devices. In 11th USENIX Symposium on Networked Systems Design and Implementation (NSDI 14). Seattle, WA, 303-316.

[11] Fabian Knutti, Mischa Sabathy, Marco Driusso, Heinz Mathis, and Chris Marshall. 2015. Positioning using LTE signals. In Proceedings of Navigation Conference in Europe. 1-8.

[12] Xiang Li, Daqing Zhang, Jie Xiong, Yue Zhang, Shengjie Li, Yasha Wang, and Hong Mei. 2018. Training-Free Human Vitality Monitoring Using Commodity Wi-Fi Devices. Proceedings of the ACM on Interactive, Mobile, Wearable and Ubiquitous Technologies 2, 3 (2018), 121.

[13] Giulio Marin, Fabio Dominio, and Pietro Zanuttigh. 2014. Hand gesture recognition with leap motion and kinect devices. In 2014 IEEE International Conference on Image Processing (ICIP). 1565-1569.

[14] Rajalakshmi Nandakumar, Vikram Iyer, Desney Tan, and Shyamnath Gollakota. 2016. FingerIO: Using Active Sonar for Fine-Grained Finger Tracking. In Proceedings of the 2016 CHI Conference on Human Factors in Computing Systems (San Jose, California, USA) (CHI '16). 1515-1525.

[15] Kai Niu, Fusang Zhang, Yuhang Jiang, Jie Xiong, Qin Lv, Youwei Zeng, and Daqing Zhang. 2019. WiMorse: A Contactless Morse Code Text Input System using Ambient WiFi Signals. IEEE Internet of Things fournal (2019), 1-1.

[16] Kai Niu, Fusang Zhang, Jie Xiong, Xiang Li, Enze Yi, and Daqing Zhang. 2018. Boosting Fine-grained Activity Sensing by Embracing Wireless Multipath Effects. In Proceedings of the 14th International Conference on Emerging Networking EXperiments and Technologies (Heraklion, Greece) (CoNEXT '18). 139-151.

[17] Junbum Park and Sung Ho Cho. 2016. IR-UWB Radar Sensor for Human Gesture Recognition by Using Machine Learning. In 2016 IEEE 18th International Conference on High Performance Computing and Communications; IEEE 14th International Conference on Smart City; IEEE 2nd International Conference on Data Science and Systems (HPCC/SmartCity/DSS). 1246-1249.

[18] Orasa Patsadu, Chakarida Nukoolkit, and Bunthit Watanapa. 2012. Human gesture recognition using Kinect camera. In 2012 Ninth International Conference on Computer Science and Software Engineering (FCSSE). 28-32.

[19] Giovanni Pecoraro, Simone Di Domenico, Ernestina Cianca, and Mauro De Sanctis. 2017. LTE signal fingerprinting localization based on CSI. In 2017 IEEE 13th International Conference on Wireless and Mobile Computing, Networking and Communications (WiMob). 1-8.

[20] Giovanni Pecoraro, Simone Di Domenico, Ernestina Cianca, and Mauro De Sanctis. 2018. CSI-based fingerprinting for indoor localization using LTE Signals.
EURASIP fournal on Advances in Signal Processing 2018, 1 (27 Jul 2018), 49.

[21] Raja Syamsul Azmir Raja Abdullah, Asem Ahmad Salah, and Nur Emileen Abdul Rashid. 2015. Moving target detection by using new LTE-based passive radar. Progress In Electromagnetics Research 63 (2015), 145-160.

[22] Ke Sun, Ting Zhao, Wei Wang, and Lei Xie. 2018. VSkin: Sensing Touch Gestures on Surfaces of Mobile Devices Using Acoustic Signals. In Proceedings of the 24th Annual International Conference on Mobile Computing and Networking (New Delhi, India) (MobiCom '18). 591-605.

[23] Michael Van den Bergh and Luc Van Gool. 2011. Combining RGB and ToF cameras for real-time 3D hand gesture interaction. In 2011 IEEE Workshop on Applications of Computer Vision (WACV). 66-72.

[24] Raghav H. Venkatnarayan, Griffin Page, and Muhammad Shahzad. 2018. Multi User Gesture Recognition Using WiFi. In Proceedings of the 16th Annual International Conference on Mobile Systems, Applications, and Services (Munich, Germany) (MobiSys '18). 401-413.

[25] Tran Huy Vu, Archan Misra, Quentin Roy, Kenny Choo Tsu Wei, and Youngki Lee. 2018. Smartwatch-based early gesture detection 8 trajectory tracking for interactive gesture-driven applications. Proceedings of the ACM on Interactive, Mobile, Wearable and Ubiquitous Technologies 2, 1 (2018), 39.

[26] Hao Wang, Daqing Zhang, Junyi Ma, Yasha Wang, Yuxiang Wang, Dan Wu, Tao $\mathrm{Gu}$, and Bing Xie. 2016. Human Respiration Detection with Commodity Wifi Devices: Do User Location and Body Orientation Matter? In Proceedings of the 2016 ACM International Joint Conference on Pervasive and Ubiquitous Computing (Heidelberg, Germany) (UbiComp '16). 25-36.

[27] Wei Wang, Alex X. Liu, Muhammad Shahzad, Kang Ling, and Sanglu Lu. 2015 Understanding and Modeling of WiFi Signal Based Human Activity Recognition. In Proceedings of the 21st Annual International Conference on Mobile Computing and Networking (Paris, France) (MobiCom '15). 65-76.

[28] Wei Wang, Alex X. Liu, and Ke Sun. 2016. Device-free Gesture Tracking Using Acoustic Signals. In Proceedings of the 22Nd Annual International Conference on Mobile Computing and Networking (New York City, New York) (MobiCom '16). $82-94$.

[29] Dan Wu, Daqing Zhang, Chenren Xu, Hao Wang, and Xiang Li. 2017. Devicefree WiFi human sensing: From pattern-based to model-based approaches. IEEE Communications Magazine 55, 10 (2017), 91-97.

[30] Dan Wu, Daqing Zhang, Chenren Xu, Yasha Wang, and Hao Wang. 2016. WiDir Walking Direction Estimation Using Wireless Signals. In Proceedings of the 2016 ACM International foint Conference on Pervasive and Ubiquitous Computing (Heidelberg, Germany) (UbiComp '16). 351-362.

[31] Jiahui Wu, Gang Pan, Daqing Zhang, Guande Qi, and Shijian Li. 2009. Gesture Recognition with a 3-D Accelerometer. In Ubiquitous Intelligence and Computing, Daqing Zhang, Marius Portmann, Ah-Hwee Tan, and Jadwiga Indulska (Eds.) Springer Berlin Heidelberg, Berlin, Heidelberg, 25-38.

[32] Yaxiong Xie, Zhenjiang Li, and Mo Li. 2019. Precise Power Delay Profiling with Commodity Wi-Fi. IEEE Transactions on Mobile Computing 18, 6 (2019), $1342-1355$.

[33] Youwei Zeng, Dan Wu, Jie Xiong, Enze Yi, Ruiyang Gao, and Daqing Zhang. 2019 FarSense: Pushing the Range Limit of WiFi-based Respiration Sensing with CSI Ratio of Two Antennas. Proceedings of the ACM on Interactive, Mobile, Wearable and Ubiquitous Technologies 3, 3 (2019), 121

[34] Daqing Zhang, Hao Wang, and Dan Wu. 2017. Toward Centimeter-Scale Human Activity Sensing with Wi-Fi Signals. Computer 50, 1 (2017), 48-57.

[35] Fusang Zhang, Kai Niu, Jie Xiong, Beihong Jin, Tao Gu, Yuhang Jiang, and Daqing Zhang. 2019. Towards a Diffraction-based Sensing Approach on Human Activity Recognition. Proc. ACM Interact. Mob. Wearable Ubiquitous Technol. 3, 1, Article 33 (March 2019), 25 pages.

[36] Fusang Zhang, Daqing Zhang, Jie Xiong, Hao Wang, Kai Niu, Beihong Jin, and Yuxiang Wang. 2018. From fresnel diffraction model to fine-grained human respiration sensing with commodity wi-fi devices. Proceedings of the ACM on Interactive, Mobile, Wearable and Ubiquitous Technologies 2, 1 (2018), 53.

[37] Ouyang Zhang and Kannan Srinivasan. 2016. Mudra: User-friendly Fine-grained Gesture Recognition Using WiFi Signals. In Proceedings of the 12th International on Conference on Emerging Networking EXperiments and Technologies (Irvine, California, USA) (CoNEXT'16). 83-96.

[38] Zhenyuan Zhang, Zengshan Tian, and Mu Zhou. 2018. Latern: Dynamic Continuous Hand Gesture Recognition Using FMCW Radar Sensor. IEEE Sensors fournal 18, 8 (2018), 3278-3289.

[39] Chen Zhao, Ke-Yu Chen, Md Tanvir Islam Aumi, Shwetak Patel, and Matthew S Reynolds. 2014. SideSwipe: Detecting In-air Gestures Around Mobile Devices Using Actual GSM Signal. In Proceedings of the 27th Annual ACM Symposium on User Interface Software and Technology (Honolulu, Hawaii, USA) (UIST '14). 527-534.

[40] Yue Zheng, Yi Zhang, Kun Qian, Guidong Zhang, Yunhao Liu, Chenshu Wu, and Zheng Yang. 2019. Zero-Effort Cross-Domain Gesture Recognition with Wi-Fi. In Proceedings of the 17th Annual International Conference on Mobile Systems, Applications, and Services (Seoul, Republic of Korea) (MobiSys '19). 313-325. 